\title{
Track reconstruction in CMS high luminosity environment
}

\section{Christophe Goetzmann*}

The CMS collaboration

E-mail: christophe.goetzmannecern.ch

The CMS tracker is the largest silicon detector ever built, covering 200 square meters and providing an average of 14 high-precision measurements per track. Tracking is essential for the reconstruction of objects like jets, muons, electrons and tau leptons starting from the raw data from the silicon pixel and strip detectors. Track reconstruction is widely used also at trigger level as it improves objects tagging and resolution. The CMS tracking code is organized in several levels, known as iterative steps, each optimized to reconstruct a class of particle trajectories, as the ones of particles originating from the primary vertex or displaced tracks from particles resulting from secondary vertices. Each iterative step consists of seeding, pattern recognition and fitting by a kalman filter, and a final filtering and cleaning. Each subsequent step works on hits not yet associated to a reconstructed particle trajectory. The CMS tracking code is continuously evolving to make the reconstruction computing load compatible with the increasing instantaneous luminosity of LHC, resulting in a large number of primary vertices and tracks per bunch crossing. This is achieved by optimizing the iterative steps and by using new software techniques. Tracking algorithms used in CMS are described; physics and computing performances are discussed with respect to Run 1 and Run 2 physics program and within CMS future upgrades.

Technology and Instrumentation in Particle Physics 2014,

2-6 June, 2014

Amsterdam, the Netherlands

* Speaker. 


\section{The CMS tracker and the LHC conditions}

The Compact Muon Solenoid (CMS) is one of the four main experiments at the Large Hadron Collider (LHC). The detector has the shape of a $21 \mathrm{~m}$ long cylinder with a diameter of $15 \mathrm{~m}$. The three first sub-detectors, the tracker, the electromagnetic and the hadronic calorimeters are placed inside the solenoid magnet, while the muon chambers are outside.

CMS uses an orthonormal right-handed coordinate system taking the nominal interaction point as origin. The $x$ axis points toward the center of the LHC, the $y$ axis points up, and the $z$ axis lies along the beam direction. The azimutal angle $\phi$ is measured in the $x-y$ plane while the polar angle $\theta$ is measured from the positive $z$ axis. The pseudorapidity is defined as $\eta=-\log (\tan (\theta / 2))$.

The CMS tracker is a $540 \mathrm{~cm}$ long cylinder with a radius of $110 \mathrm{~cm}$, fully composed of silicon detectors. It is the innermost detector of CMS, and its purpose is the reconstruction of charged particles trajectories, from which charge and momentum are estimated. The positions of primary vertices are also estimated using tracks informations.

The inner part of the tracker, closer to the collision point, is composed of pixel detectors. They are organised in 3 concentric cylinders at radius $4.4,7.3$ and $10.2 \mathrm{~cm}$, completed by 2 endcap disks on each side. Each pixel has a size of $100 \times 150 \mu \mathrm{m}^{2}$ and uses silicon of $285 \mu \mathrm{m}$ thickness. The signal from pixel is read by a Read Out Chip (ROC), each ROC being attached to an array of $80 \times 52$ pixels.

The rest of the tracker is composed of microstrip detectors, organised in two barrels (TIB and TOB for a total of 10 layers) and two types of endcap disks (TID and TEC for a total of 12 layers on each side). A schematic representation of the tracker is presented in Figure 1. Unlike the pixels, microstrip detectors have different characteristics depending on the region were they are placed. The silicon thickness varies from 320 to $500 \mu \mathrm{m}$ and the pitch from 80 to $205 \mu \mathrm{m}$. On some layers, pairs of microstrip detectors are glued back-to-back with a relative angle of $100 \mathrm{mrad}$, in order to provide a two dimensional information. These are referred to as stereo layers. The fiducial acceptance of the tracker corresponds to the region $|\eta|<2.5$ [1].

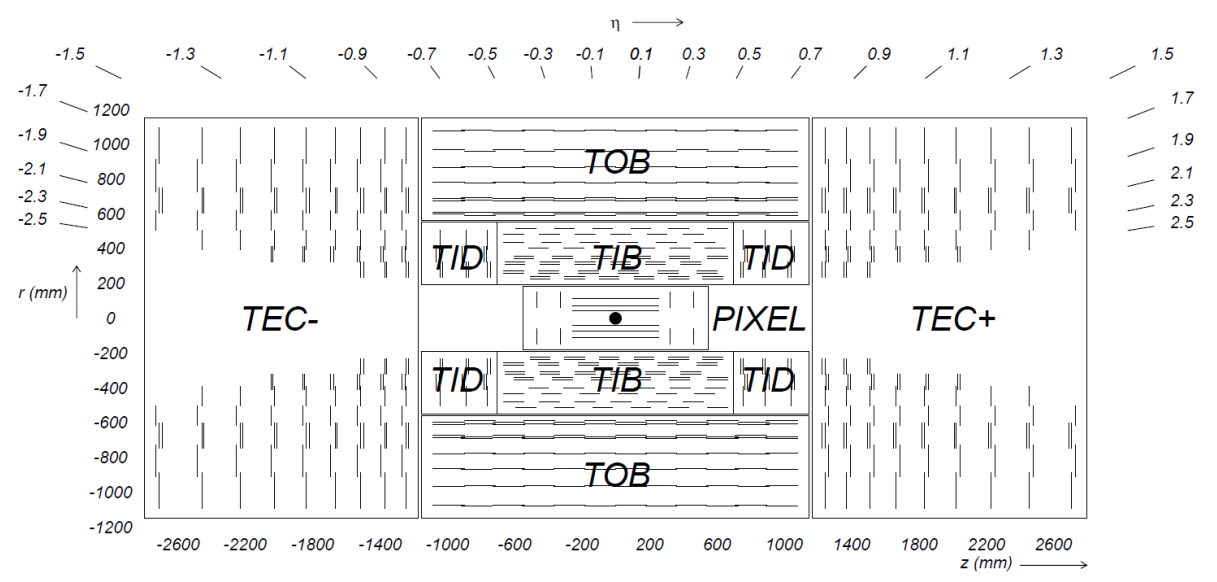

Figure 1: Schematic cross section through the CMS tracker in the $r-z$ plane, with $r=\sqrt{x^{2}+y^{2}}$. Double lines indicate back-to-back silicon strip modules that provide stereo hit position measurement. 
Being the innermost sub-detector of CMS, the tracker has to face a high flux density. This flux is proportionnal to the instantaneous luminosity. At the nominal luminosity of $10^{34} \mathrm{~cm}^{-2} \mathrm{~s}^{-1}$, the particles flux at $4.4 \mathrm{~cm}$ from collision point is close to $40 \mathrm{M} / \mathrm{cm}^{2} / \mathrm{s}$. The peaks of instantaneous luminosity delivered by the LHC from 2010 to 2012 is shown in Figure 2 [3]. The maximum reached in 2012 corresponds to an average number of 25 proton-proton interactions per bunch crossing. We refer to these additional collisions as pile-up interactions. This high occupancy constitutes a challenge for both hit and track reconstruction.

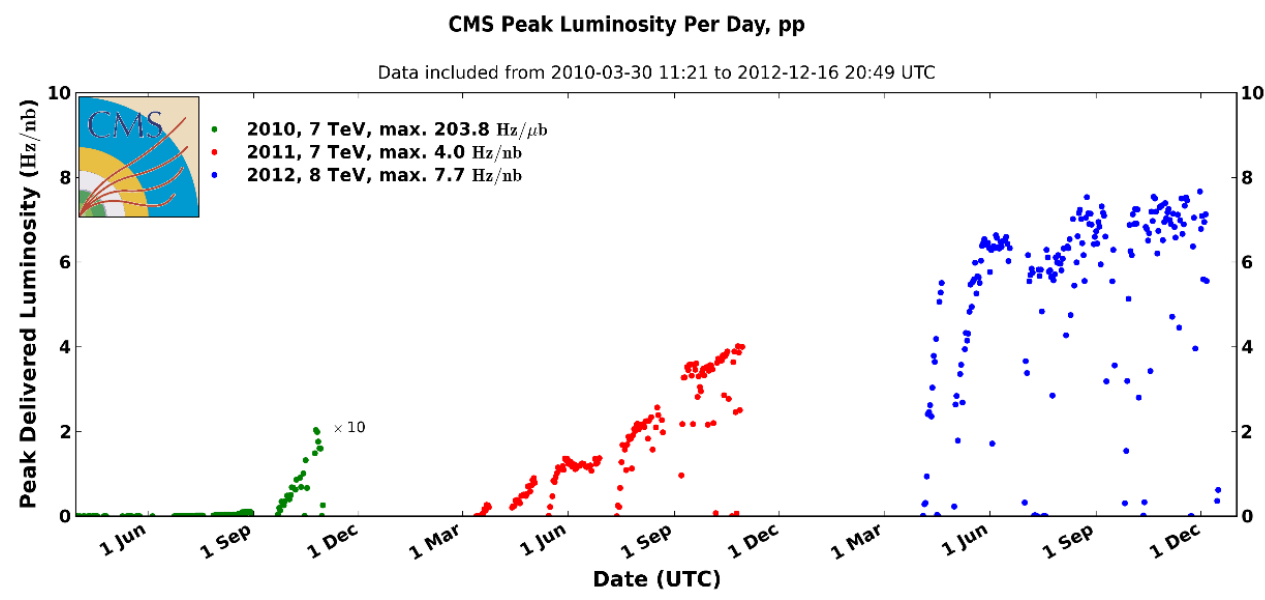

Figure 2: Instantaneous luminosity for proton-proton collisions delivered by the LHC from 2010 to 2012 (Run I).

The level of radiation recieved by sensors over time also affects the performances of the silicon detectors, and its influence has to be taken into account. During LHC Run I, proton-proton collisions were performed at an energy of 7 and $8 \mathrm{TeV}$ in the center of mass. The total amount of collisions produced by the LHC in CMS, expressed as integrated luminosity, was approximately $30 \mathrm{fb}^{-1}$.

\section{Hit reconstruction}

The track reconstruction starts with the reconstruction of hits by the various sensitive layers of the tracker. For the microstrip detectors, the signal from each channel is read by an analog chip (APV25), and sent to an electronic board that substracts pedestals and performs zero suppression. Starting from any channel with a signal to noise ratio above 3, adjacent channels with a signal to noise ratio above 2 are grouped into clusters. The hit position is estimated via a charge-weighted mean of the strip positions. A correction is applied to account for the Lorentz drift of the collected charges in the CMS magnetic field (10 $\mu \mathrm{m}$ in TIB, $20 \mu \mathrm{m}$ in TOB).

For the pixel detectors, adjacent pixels (having a common corner or side) with a charge above a certain configurable threshold are grouped into clusters. The charge from all pixels is then projected onto two local $u$ and $v$ perpendicular axis. Projected clusters are obtained, that are used for 
the position estimation on transverse and longitudinal direction. There are two independant techniques used to estimate this hit position. The first-pass hit reconstruction calculate the position on $u$ and $v$ by adjusting the geometrical center of the projected cluster, taking into account the charges of its first and last pixels, and its geometrical width [2]. Eventually, an additional correction is applied to account for Lorentz drift, in the transverse plan. This algorithm is fast, and is used in track seeding and track finding steps, described in the next section. For a pixel detector that has not been exposed to radiations, the resolution in the transverse plane $(r-\phi)$ should be of the order of $10 \mu \mathrm{m}$.

The pixel detectors in CMS are close to the interaction point and face high radiation level. The first pass hit reconstruction is not robust against damages due to radiation. Indeed, for strongly irradiated sensors, the resolution is significantly degraded and the hit position estimation can be biased by up to $50 \mu \mathrm{m}$. For this reason, a complementary template based reconstruction algorithm is used for the final track fit described in the next section. In this method, the measured cluster charge distribution is compared to a collection of templates distributions, obtained via simulation. These templates are produced using the Pixelav program [4] [5] [6]. In particular, the effect of radiation on sensors can be simulated, and therefore, the evolution of cluster charge distribution over time can be anticipated. This way, a good resolution, better than $10 \mu \mathrm{m}$ in the transverse plane, can be maintained through the full period of data taking.

The resolution of pixel detectors is also affected by the increase of the thresholds and the analog current in the ROCs as a function of integrated luminosity. These thresholds are regulary measured with charge injection. The results of these measurements in 2011 and 2012 are presented in Figure 3. An increase in the thresholds implies a reduction of the average cluster charge and size, resulting in a degraded resolution. To control this effect, calibrations are performed during technical stops, and the thresholds are adjusted. The drops observed in the distributions of Figure 3 are the consequence of these adjustments [7].

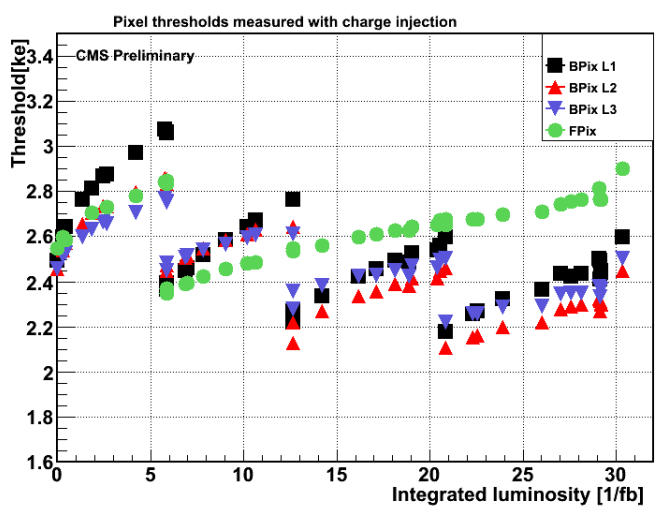

Figure 3: Average pixel thresholds in unit of 1000 electrons as a function of delivered integrated luminosity for the 3 barrel layers (in black, red, and blue) and the forward pixel disks (in green).

Pixel performances are also sensible to instantaneous luminosity. First of all, with higher occupancy, the power consumption, and therefore the temperature of the ROCs, is increased, influ- 
encing the charge value in output. Furthermore, the internal buffer of the ROCs are more frequently filled, and the overflow probability is higher. And finally, particles produced in the collisions can eventually flip bits in ROCs and auxiliary electronics, leading to degradation or interruption of data taking, with a probability increasing with occupancy. The hit efficiency of the different pixel layer with respect to instantaneous luminosity is presented in Figure 4 [7].

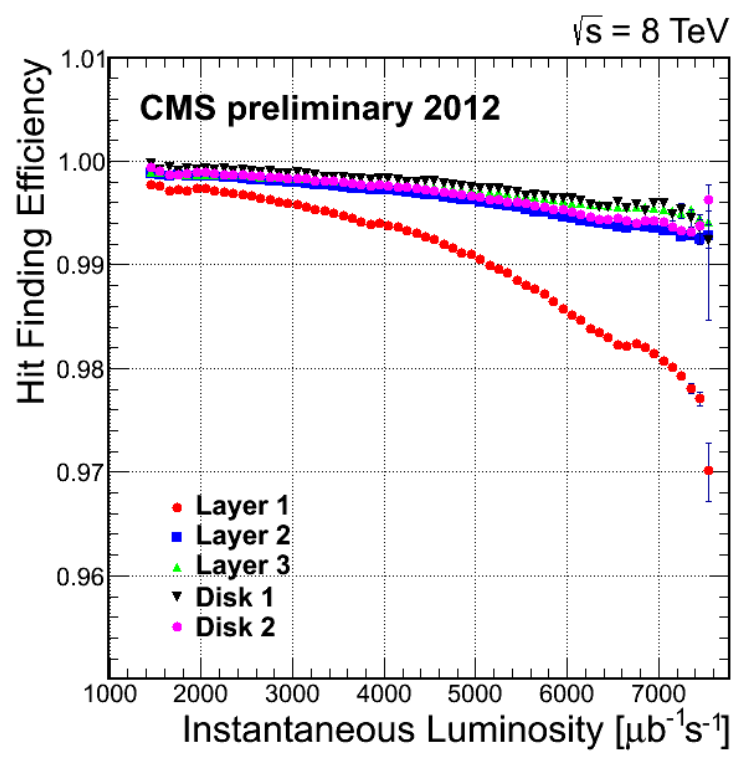

Figure 4: Hit reconstruction efficiency in 2012 data for the different pixel layers as a function of the delivered instantaneous luminosity.

\section{Track reconstruction and iterative tracking optimization}

The main challenge associated to track reconstruction in the CMS tracker is related to the large number of hits produced at each bunch crossing. The reconstruction proceeds in 4 steps:

- Seed generation: a first estimation of track parameters using a minimal combination of hits (3 hits, or 2 hits with a constraint on vertex position) is performed.

- Track finding: propagate the seed through the tracker, and use a Kalman filter based technique to find compatible hits to be associated with the building track. The track is propagated layer by layer, the parameters being updated after each step. If more than one compatible hit is found on a given layer, several tracks are built in parallel. For this reason, a procedure called ambiguities resolution is run once the track finding is over. It consists in comparing all pairs of tracks and, if their fraction of common hits is above $19 \%$, selecting only the best track based on the number of hits and $\chi^{2}$.

- Track final fit: using all the associated hits, a fit of the trajectory is performed to obtain a more precise estimation of track parameters. It uses a Kalman filter and a smoother algorithm. 
- Track selection: quality requirements are applied. The tracks are categorized in different quality collections, according to the set of criteria they fullfill: loose, tight, or high purity. Tracks that do not pass any selection are discard. The goal of this selection step is to further reduce the fake rate.

The particularity of the CMS tracking software is that these four steps are run not only once, but several times, in a process called iterative tracking. Each iteration is defined by the criteria used for the seed generation: type of hits, number of hits, $p_{T}$ cuts, and impact parameters cuts. The different iterations are run successively, and after an iteration is over and has led to the successful reconstruction of a certain number of tracks, all the hits associated to these tracks are masked in the hit collection, and the next iteration begins on a reduced number of hits.

The tracking software is fully configurable. The characteristics of each iteration can be changed, and new iterations can eventually be added to optimize the tracking performances, especially in terms of CPU time. As a matter of fact, in 2011, it appeared that the first version of tracking software was not performing well enough to ensure satisfying prompt reconstruction performances with the rising number of pile-up events expected for 2012. During the Run I of the LHC, two improvement campaigns have therefore been conducted.

The first campaign, in fall 2011, was implemented on the version 4.4.X of the official CMS software (CMSSW). It consists firstly in a set of modifications in the code itself, with a better use of the memory resources and smarter algorithms, that does not affect the physics outcome. These modifications resulted in a $40 \%$ cut of the memory budget. The details of these modifications can be found in [8]. In a second pass, the configuration of the different iterations has also been optimized. In Tables 1 and 2 the configurations before and after improvements are shown. The effect of these changes has been tested with Monte Carlo QCD events with 30 pile-up interactions. The CPU time dedicated to tracking is found to be reduced by a factor 2.5. The modifications of the thresholds affect the observables, but the effect on performances are minor with respect to physics outcome.

\begin{tabular}{|c|c|c|c|c|c|}
\hline Iteration & seed type & seed subdetectors & $p_{T}[\mathrm{GeV} / \mathrm{c}]$ & $d_{0} \mathrm{cut}$ & $z_{0} \mathrm{cut}$ \\
\hline 0 & triplet & pixel & $>0.8$ & $0.2 \mathrm{~cm}$ & $3.0 \sigma$ \\
1 & pair & pixel/TEC & $>0.6$ & $0.05 \mathrm{~cm}$ & $0.6 \mathrm{~cm}$ \\
2 & triplet & pixel & $>0.075$ & $0.2 \mathrm{~cm}$ & $3.3 \sigma$ \\
3 & triplet & pixel/TIB/TID/TEC & $>0.25-0.35$ & $2.0 \mathrm{~cm}$ & $10.0 \mathrm{~cm}$ \\
4 & pair & TIB/TID/TEC & $>0.5$ & $2.0 \mathrm{~cm}$ & $12.0 \mathrm{~cm}$ \\
5 & pair & TOB/TEC & $>0.6$ & $6.0 \mathrm{~cm}$ & $30.0 \mathrm{~cm}$ \\
\hline
\end{tabular}

Table 1: Configuration of the different tracking iterations before the first improvement campaign in fall 2011. $d_{0}$ and $z_{0}$ are respectively the transverse (in $x-y$ plane) and longitudinal impact parameters. $\sigma$ corresponds to the width of the beam spot along the $z$ axis.

A second improvement campaign followed in 2012, with further enhancement of the code, including a change of compiler and ROOT versions. The iterative tracking was again slightly 


\begin{tabular}{|c|c|c|c|c|c|}
\hline Iteration & seed type & seed subdetectors & $p_{T}[\mathrm{GeV} / \mathrm{c}]$ & $d_{0}$ cut & $z_{0}$ cut \\
\hline 0 & triplet & pixel & $>\mathbf{0 . 6}$ & $\mathbf{0 . 0 3} \mathrm{cm}$ & $\mathbf{4 . 0} \sigma$ \\
1 & triplet & pixel & $>\mathbf{0 . 2}$ & $\mathbf{0 . 0 3} \mathrm{cm}$ & $\mathbf{4 . 0} \sigma$ \\
2 & pair & pixel & $>0.6$ & $\mathbf{0 . 0 1} \mathrm{cm}$ & $\mathbf{0 . 0 9} \mathrm{cm}$ \\
3 & triplet & pixel & $>\mathbf{0 . 2}$ & $\mathbf{1 . 0} \mathrm{cm}$ & $\mathbf{4 . 0} \sigma$ \\
4 & triplet & pixel/TIB/TID/TEC & $\mathbf{> 0 . 3 5 - 0 . 5 0}$ & $2.0 \mathrm{~cm}$ & $10.0 \mathrm{~cm}$ \\
5 & pair & TIB/TID/TEC & $>\mathbf{0 . 6}$ & $2.0 \mathrm{~cm}$ & $\mathbf{1 0 . 0} \mathrm{cm}$ \\
6 & pair & TOB/TEC & $>0.6$ & $6.0 \mathrm{~cm}$ & $30.0 \mathrm{~cm}$ \\
\hline
\end{tabular}

Table 2: Configuration of the different tracking iterations after the first improvement campaign in fall 2011. A new pixel triplet iteration has been added. The parameters that have been changed with respect to the previous version are in bold.

modified with respect to the configuration presented in table 2. No major changes were needed, the fall 2011 configuration being already quite performant even for the maximum level of pile-up reached in 2012. The details of this second improvement campaign can be found in [8]. With respect to the baseline configuration, the overall gain is about a factor 2 reduction of memory load and factor 7 reduction of CPU time for typical 2012 events at 20 pile-up interactions.

Finally, the flexibility of the tracking software also enables the add of new iterations dedicated to the reconstruction of particular types of tracks. For instance, two new iterations dedicated to the reconstruction of muon tracks have been added in 2014. The first one uses tracks in the muon system as seeds, and the track reconstruction is performed outside-in, starting from this seed. A second iteration consists in a re-reconstruction of tracker tracks that have been matched to a track in the muon chambers, but with looser requirements in the track finding step, in order to collect possible missing hits. The gain in term of muon tracking efficiency is shown in Figure 5, with respect to the late 2012 configuration without these new iterations. These plots have been obtained from data, using a Tag and Probe method, described in [9].

\section{Conclusions}

In 2013, CMS ended a period of data taking that has lasted 3 years, with collsions at $\sqrt{s}=7$ and $8 \mathrm{TeV}$. Performant prompt reconstruction efficiencies have been maintained despite the increasing instantaneous luminosity, thanks to a very efficient hit reconstruction (above $99 \%$ on average), and the crucial improvements in tracking software described in this paper.

Nevertheless, the LHC will start again in 2015, with a significantly increased luminosity and energy, possibly leading to a much higher level of pile-up. Together with the phase 1 improvement of the tracker (new pixel detector in 2016), additional software improvements will be needed. The possibility of adding new tracking techniques, like Hough transforms tracking, are studied. Parallelization of the code is also being considered, taking advantage of the increasing number of CPU cores. 

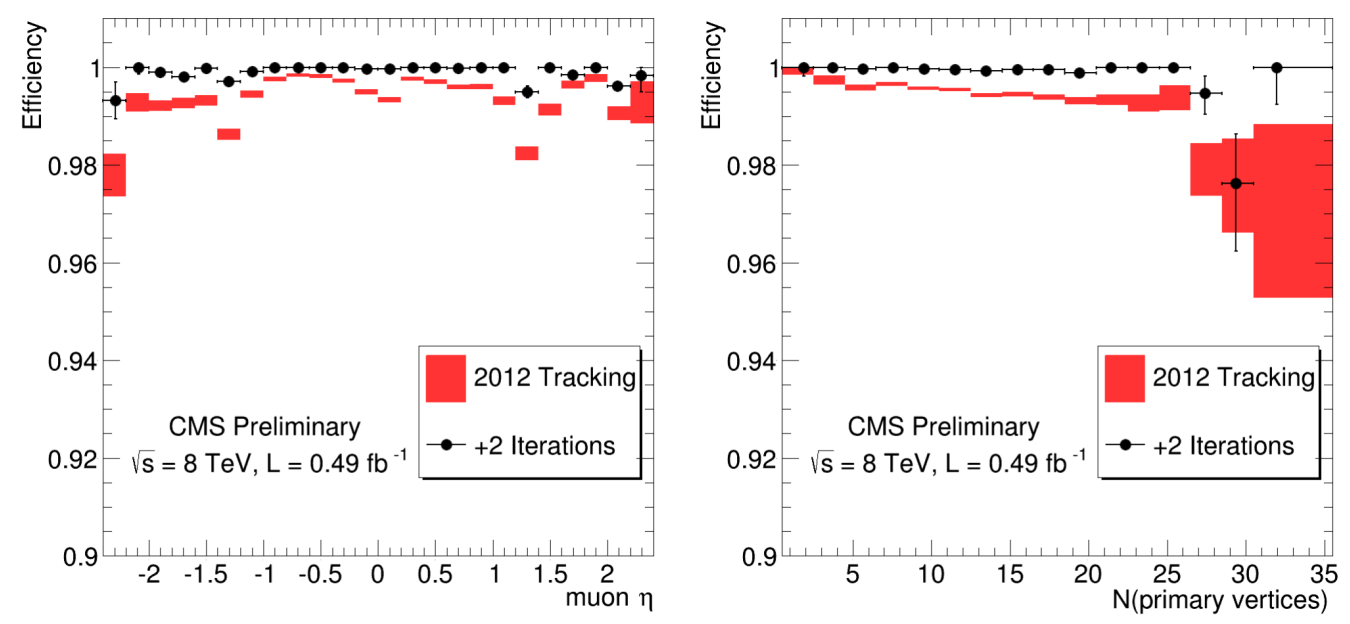

Figure 5: Efficiency of muon track reconstruction in data with and without the 2 new muon iterations, estimated from a Tag and Probe method in 2012 data. The efficiency is shown with respect to pseudorapidity (left), and pile-up (right).

\section{References}

[1] The CMS Collaboration, The CMS experiment at the CERN LHC, JINST 3 (2008) S08004 [doi:10.1088/1748-0221/3/08/s08004]

[2] The CMS Collaboration, Description and performance of track and primary vertex reconstruction with the CMS tracker, [arXiv:1405.6569]

[3] The CMS Collaboration, CMS Luminosity - Public Results, [https://twiki.cern.ch/twiki/bin/view/CMSPublic/LumiPublicResults ]

[4] M. Swartz, CMS pixel simulations, Nucl.Instrum.Meth A511 (2003) 88-91 [doi:10.1016/S0168-9002(03)01757-1]

[5] V. ChioChia et al., Simulation of Heavily Irradiated Silicon Pixel Sensors and Comparison With Test Beam Measurements, IEEE Trans. Nucl. Sci 52 (2005) 1067-1075 [arXiv: physics / 0 41143]

[6] M. Swartz, Observation, modeling, and temperature dependence of doubly peaked electric fields in irradiated silicon pixel sensors, Nucl.Instrum.Meth A565 (2006) 212-220

[arXiv:physics/0510040]

[7] The CMS Collaboration, CMS Tracker Detector Performance Results, [https://twiki.cern.ch/twiki/bin/view/CMSPublic/PixelofflinePlots2013 ]

[8] G. Sguazzoni and D. Giordano, CMS reconstruction improvements for the tracking in large pile-up events, Journal of Physics: Conference Series 396 (2012) 022044 [doi:10.1088/1742-6596/396/2/022044]

[9] The CMS Collaboration, Performance of CMS muon reconstruction in pp collision events at $\sqrt{s}=7$ TeV, JINST 7 (2012) P10002 [arXiv: 1206.4071$]$

[10] R. Brun and F. Rademakers ROOT âĂ $\breve{T}$ An object oriented data analysis framework Nucl.Instrum.Meth A389 (1997) 81-86 [doi:10.1016/S0168-9002 (97) 00048-X] 